\title{
Calculation of the Doppler broadening of the electron-positron annihilation radiation in defect-free bulk materials
}

\author{
V. J. Ghosh \\ Brookhaven National Laboratory, Upton, New York 11973 \\ M. Alatalo \\ Helsinki Institute of Physics and Laboratory of Physics, P.O. Box 1100, 02015, University of Helsinki, Helsinki, Finland \\ P. Asoka-Kumar \\ Lawrence Livermore National Laboratory, Livermore, California 94550 \\ B. Nielsen \\ Brookhaven National Laboratory, Upton, New York 11973 \\ K. G. Lynn \\ Materials Research Center, Washington State University, Pullman, Washington 99613 \\ A. C. Kruseman ${ }^{*}$ and P. E. Mijnarends \\ Interfaculty Reactor Institute, Delft University of Technology, $2629 \mathrm{JB}$ Delft, The Netherlands
}

(Received 27 April 1999; revised manuscript received 19 November 1999)

\begin{abstract}
Results of a calculation of the Doppler broadening of the positron-electron annihilation radiation and positron lifetimes in a large number of elemental defect-free materials are presented. A simple scheme based on the method of superimposed atoms is used for these calculations. Calculated values of the Doppler broadening are compared with experimental data for a number of elemental materials, and qualitative agreement is obtained. These results provide a database which can be used for characterizing materials and identifying impurityvacancy complexes.
\end{abstract}

\section{INTRODUCTION}

Positron annihilation spectroscopy is a well-established tool for studying defects in solids. ${ }^{1-3}$ In a defect-free crystal the positrons experience a periodic repulsive potential centered on the ion cores. Open-volume defects and negative charge centers form isolated minima in the periodic potential, and act as positron traps. This propensity of positrons to trap selectively at open-volume defects has made them an ideal probe for looking at vacancies, vacancy clusters, and vacancy-impurity complexes. The trapped positrons have a longer lifetime than untrapped or delocalized positrons. Eventually all positrons annihilate with electrons predominantly via two gamma ray decay. Measurements of the positron lifetime and the Doppler broadening of the annihilation radiation have been used to study and identify defects in metals, semiconductors, and polymers. ${ }^{3}$

The Doppler broadened annihilation $\gamma$-ray spectrum is the sum total of the contributions of annihilations with different electron states at the annihilation site. Annihilations with delocalized and weakly bound valence electrons contribute to the low-momentum part of the momentum distribution, whereas tightly bound core electrons contribute to the highmomentum part. Traditional one-detector Doppler broadening measurements have been used to study line-shape variations in the low- and intermediate-momentum regions. However, line-shape variations due to the core electrons in the high-momentum regime cannot be measured easily by that technique because the signal-to-noise ratio is insufficient. When a coincidence measurement of the energies of both the $\gamma$ rays is made using two similar detectors the signal to background ratio can be improved from about 100 to 1 to $10^{5}$ to $1 .{ }^{4,5}$ This allows one to make accurate measurements of both the valence and core electron contributions to the Doppler broadening up to $p_{L}=\left(60 \times 10^{-3}\right) m_{0} c$, where $p_{L}$ is the momentum component along the direction of the $\gamma$-ray emission. It has been shown earlier that the two-detector technique can be used to obtain enhanced elemental specificity ${ }^{4}$ and to observe the effect of lattice structure on the Doppler broadening. ${ }^{6}$ This technique has been used in the study of defects in metals and semiconductors. ${ }^{7-10}$ For a unique interpretation of the experimental results in these often complicated systems, it is useful to have a comprehensive set of calculations for different elements which can be used to identify, or exclude, the presence of certain types of atoms at the annihilation site.

In this paper we present results of Doppler broadening calculations based on the method of superimposed atoms for a large number of materials. Some of these results are compared with experimental data. Since positron lifetimes are calculated simultaneously, they are also compared with existing experimental data. The computational method used is described in Sec. II. Section III contains our Doppler broadening results and comparisons with representative experimental data. Comparisons of calculated and measured positron lifetimes are also presented in this section. A summary of the work and conclusions is presented in Sec. IV. 


\section{COMPUTATIONAL METHOD}

In our calculations, we have used the method of Alatalo and co-workers ${ }^{7,11}$ for computing the momentum distribution of the annihilating electron-positron pairs. This method is based on a Jastrow-like approximation for the momentum distribution for each electron state $i$,

$$
\rho_{i}(\mathbf{p})=\pi r_{e}^{2} c u_{i}^{2}(0)\left|\int d \mathbf{r} e^{\mathrm{i} \mathbf{p} \cdot \mathbf{r}} \psi_{+}(\mathbf{r}) \psi_{i}(\mathbf{r})\right|^{2},
$$

where $\psi_{+}(\mathbf{r})$ and $\psi_{i}(\mathbf{r})$ are the single-particle wave functions for the positron and the electrons, respectively, and $u_{i}^{2}(0)$ are the state-dependent enhancement factors. $r_{e}$ and $c$ are the classical electron radius and the speed of light, respectively. The state-dependent enhancement factors $u_{i}^{2}(0)$ are calculated using the partial annihilation rates for the state $i{ }^{11}$ This is a reasonable approximation for the core electron states, but does not necessarily hold for the delocalized conduction electrons. In what follows, we are mainly interested in the annihilations with the core electrons, and therefore the approximation for the state-dependent enhancement factors can be assumed to be valid. One should also note that for this reason the comparison of our calculations with the experiments is not meaningful below $\simeq 20 \mathrm{mrad}$. In order to achieve quantitative agreement with the experiments in the low-momentum region, one should perform a self-consistent band calculation for the valence electrons. ${ }^{12-14}$ This is, of course, computationally much more demanding, which limits the use of such methods to cases where an accurate calculation for one or very few different systems is needed. In order to obtain trends, however, it is preferable to use simpler methods, such as the present one.

In the calculation of the positron wave functions and the ensuing annihilation rates and lifetimes we used the atomic superposition (AS) method. ${ }^{15,16}$ The annihilation rates and enhancement factors were calculated using the generalized gradient approximation (GGA) introduced by Barbiellini et al. ${ }^{17}$. The GGA has been shown to yield good values for the positron lifetimes in a wide range of elements and semiconducting compounds. ${ }^{17}$ This agreement is partly due to the well-chosen value for the parameter $\alpha$ in the expression for the GGA enhancement factor. ${ }^{17}$ Indeed, the lifetimes change almost linearly as a function of this parameter. Our test calculations for $\mathrm{Si}$, however, indicate that the value of $\alpha$ has very little effect on the shape of the momentum distribution.

The effect of using different electron wave-function programs was explored and no significant differences were found for the lighter elements. ${ }^{18}$ However, for $\mathrm{W}$, significant differences were observed between relativistic and nonrelativistic electron wave functions, and therefore the relativistic electron wave functions were used for the momentum density overlap integrals. ${ }^{19,20}$ The calculations reported in this paper were performed using positron wave functions obtained from the atomic superposition model for evaluating the annihilation rates, the overlap integrals, and the enhancement factors. The AS positron wave functions depend on the crystal geometry and exhibit anisotropy. The results quoted in the paper use spherically averaged positron wave functions. Doppler profiles are a one-dimensional (1D) projection of the 3D momentum density, and this projection smoothes out some of the anisotropy. Furthermore, convolution of the detector resolution function with the calculated profile smoothes the results even further. No differences were observed for Doppler profiles in Al for different crystal orientations $\left([001]\right.$ and [114]). ${ }^{21}$

The major motivation for using AS positron wave functions was to check whether they provide a realistic description. The linear-muffin-tin-ovbital (LMTO) method can be used to calculate more accurate positron wave functions in defect-free bulk materials, but it is extremely difficult to do so for large supercells containing multiple vacancies or impurity-vacancy clusters. Even in systems containing defects, AS wave funtions can be calculated easily. Whether these wave functions provide a realistic description can only be determined by detailed comparisons between experimental and calculated results. To compare the experimental and theoretical results the calculated momentum distributions were convoluted with a Gaussian function (of a half-width $1.5 \mathrm{keV}$ ) to include the detector resolution, and both distributions were normalized to unit area.

\section{RESULTS}

Doppler broadening measurements have been performed on a number of defect-free bulk materials. Comparisons between calculations (using LMTO positron wave functions) and experimental data for diamond, $\mathrm{Al}, \mathrm{Si}, \mathrm{Fe}, \mathrm{Ni}, \mathrm{Cu}, \mathrm{Ge}$, $\mathrm{Sn}$, and $\mathrm{W}$ were shown in Ref. 4. These results show qualitative agreement between the two. Calculations using the atomic superposition method have been done for a large number of materials: C, Al, Si, Fe, Ni, Cu, Zn, Ge, Nb, Mo, $\mathrm{Pd}, \mathrm{Ag}, \mathrm{Sn}, \mathrm{La}, \mathrm{Ce}, \mathrm{Eu}, \mathrm{Gd}, \mathrm{Yb}, \mathrm{W}, \mathrm{Au}$, and Pb. Calculations for $\mathrm{C}$ were done for both diamond and graphite crystal structures, and those for Sn for $\alpha$ and $\beta \mathrm{Sn}$. These results were discussed in Ref. 6.

The calculated momentum distributions for $\mathrm{Al}, \mathrm{Cu}, \mathrm{Nb}$, and $\mathrm{W}$ have been compared with the experimental data in Figs. 1, 2, 3, and 4. In these figures the contributions of both valence and core electrons to the Doppler broadening have been included, even though the valence electron contribution had not been included in earlier work..$^{4,7,11}$ The argument for leaving the valence contribution out earlier was that this model did not provide a good description of valence electrons, and hence their contribution should not be included. This is true in the present case as well, and the comparison between experiment and theory is not reliable below about $\left(20 \times 10^{-3}\right) m_{0} c$. However, in spite of the fact that this is a very crude approximation for the valence electrons, it still gives qualitative agreement with experimental data, and can be used for studying trends and making qualitative comparisons.

In these figures we have shown the total Dopplerbroadened line shapes as well as the individual contributions due to annihilations with different electron levels. Since we use relativistic electron wave functions, the $p, d$, and $f$ levels are split into two levels corresponding to different total angular momentum $j$. Their contributions are qualitatively the same, and follow the same trends. The purpose of showing these individual contributions is to illustrate which electrons make the dominant contribution in a given momentum re- 


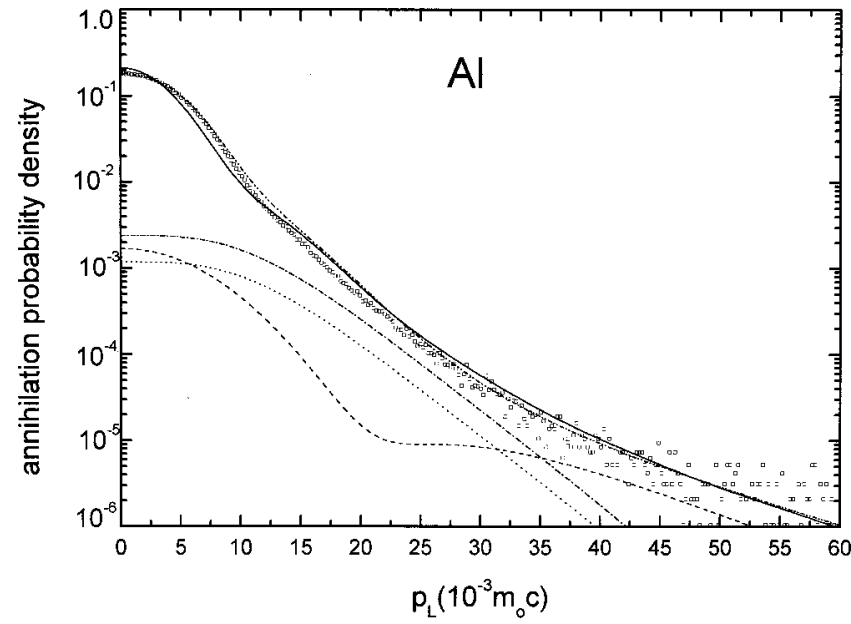

FIG. 1. Comparison of the calculated (solid line) and experimental (open squares) annihilation probability densities for Al. The contributions of the different electron levels have also been plotted in the same figure. The area under the curve has been normalized to unity for both the experimental and calculated results. The contribution of the $2 s_{1 / 2}$ electrons (dashed line), the $2 p_{1 / 2}$ electrons (dotted line), and the $2 p_{3 / 2}$ electrons (dot-dashed line) electrons are also shown. The dot-dot-dashed line represents the results of a KKR band-structure calculation (Ref. 14).

gion. As we see by comparing these figures, the same electron level does not make the dominant contribution to a specific momentum region for different atoms. We also note that qualitative agreement between the calculated and experimental results is obtained over the whole momentum range, although all four curves have regions where the disagreement is as large as a factor of 2 .

The detector resolution has a significant impact on the shape and width of the Doppler profiles. ${ }^{22}$ The importance of the detector resolution had been largely unappreciated when these experiments were first started, and it was not consid-

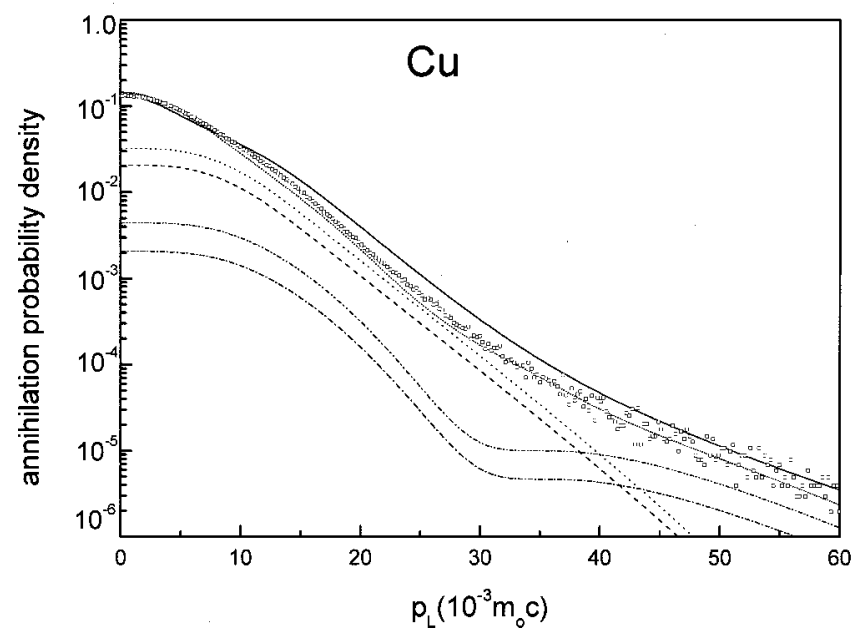

FIG. 2. Comparison of the calculated (solid line) and experimental (open squares) annihilation probability densities for $\mathrm{Cu}$. The contributions of $3 d_{3 / 2}$ (dashed line), $3 d_{5 / 2}$ (dotted line), $3 p_{1 / 2}$ (dotdashed line), and the $3 p_{3 / 2}$ (dot-dot-dashed line) electrons are also shown. The small dashed line is the result of a LMTO-ASA bandstructure calculation.

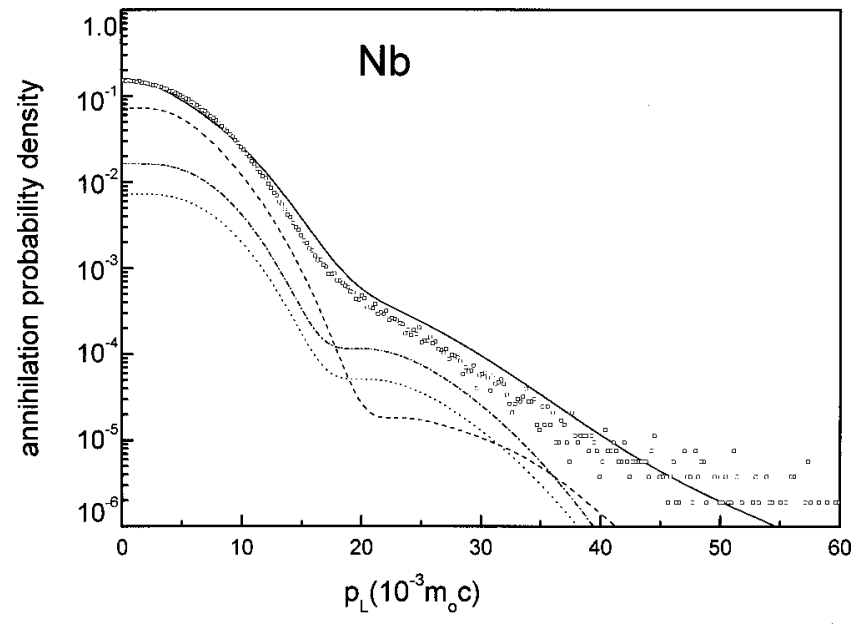

FIG. 3. Comparison of the calculated (solid line) and experimental (open squares) annihilation probability densities for $\mathrm{Nb}$. The contributions of the $4 d_{3 / 2}$ (dashed line), $4 p_{1 / 2}$ (dotted line), and $4 p_{3 / 2}$ electrons ( dot-dashed line) are also shown.

ered necessary to measure and report the detector resolution, especially when reporting measurements in the momentum region $\left[(20-50) \times 10^{-3}\right] m_{0} c$. The results of Ref. 22 compare Doppler profiles corresponding to different values of the detector resolution [the full width at half maximum (FWHM) of the detector resolution was varied from 0.9 to $2.5 \mathrm{keV}$ ], and show that the width of the Doppler profiles increases with the width of the resolution of the detectors used for the measurements. The impact of the change is largest at small momenta but it appears to extend to about $\left(50 \times 10^{-3}\right) m_{0} c$. This implies that the resolution influences the results not only of the valence electron-positron annihilations but of the core electron annihilations as well.

In Fig. 1 for bulk $\mathrm{Al}$ we see that the contributions of the $3 s$ and $3 p$ valence levels dominate at low momenta, namely below $\left(15 \times 10^{-3}\right) m_{0} c$. The contribution of the $2 p$ levels is dominant up to $35 m_{0} c$, after which the $2 s$ level is dominant. The $1 s$ level becomes the dominant contribution above our

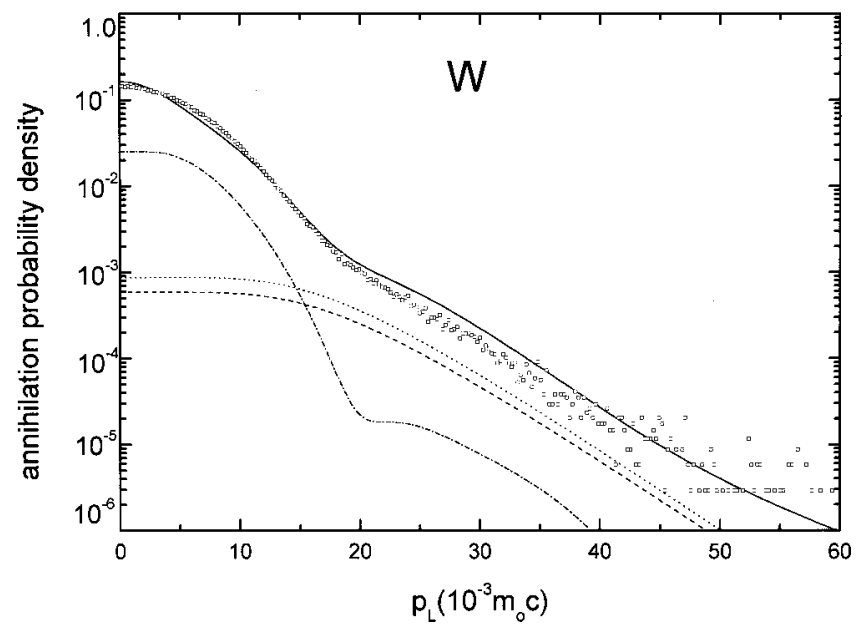

FIG. 4. Comparison of the calculated (solid line) and the experimental (open squares) annihilation probability densities for $\mathrm{W}$. The contributions of $5 d_{3 / 2}$ (dot-dashed line), the $4 f_{5 / 2}$ (dashed line), and $4 f_{7 / 2}$ (dotted line) electrons are also shown. 
experimental range. From this figure, it would appear that the use of free-atom electron wave functions to represent valence electrons causes the disagreement between calculated and experimental results. The agreement between calculated and experimental values improves when the positron and electron wave functions are calculated using the Korringa-KohnRostoker (KKR) method, and the local-densityapproximation is used for the enhancement factors. ${ }^{14}$ These results are represented in Fig. 1 by the dot-dot-dashed line, and show improved agreement in all except the $[(10-20)$ $\left.\times 10^{-3}\right] m_{0} c$ region.

The calculated momentum distribution for $\mathrm{Si}$ was compared earlier to experimental data, ${ }^{4}$ and the calculated values were found to be lower than the experimental values for the entire momentum range. This is probably due to the fact that the effect of covalent bonding is not included in the AS model. If covalent bonding were included, it would decrease the screening of the core electrons and increase the core contribution, giving better agreement with the experimental results. A self-consistent calculation for the positron lifetime in defect-free materials ${ }^{23}$ yields good agreement with experimental data. Quantitative agreement with experiment can also be obtained for open-volume defects in Si using a similar approach. ${ }^{13}$ Pseudopotential methods have also been used to calculate positron lifetimes ${ }^{24}$ and momentum distributions, ${ }^{25}$ and good agreement with experiments is obtained.

In Fig. 2 the calculated results for $\mathrm{Cu}$ are compared with the experimental data. Similar comparisons were made for $\mathrm{Ni}, \mathrm{Fe}$, and Ge. Excellent agreement was obtained for $\mathrm{Ge}^{4}$ Some disagreement was observed between calculated and experimental values in the $\left[(15\right.$ to 40$\left.) \times 10^{-3}\right] m_{0} c$ range for $\mathrm{Fe}, \mathrm{Ni}$, and $\mathrm{Cu}$. Looking at the curves for different electron levels we see that in this region the dominant contribution is due to the $3 d$ electrons, and this contribution is being overestimated by our calculations. In transition elements like $\mathrm{Cu}$, $\mathrm{Fe}$, and $\mathrm{Ni}$, the $3 d$ electrons form bands and are quite delocalized in real space. If this fact were taken into account it would limit their momentum space contribution to smaller momenta, thereby giving better agreement with experiment. Results of full band structure calculations using the LMTO method with the atomic-sphere approximation (ASA), and state-dependent enhancement factors within the GGA (Ref. 26) are shown in Fig. 2 by the small dashed curve. This calculation demonstrates better agreement with the experimental data than the free-atom calculation.

The agreement between the calculated and experimental results in the case of Ge (Ref. 4) may be due to the partial cancellation of two errors. Since the effect of covalent bonding and the resultant screening of the $3 d$ electrons by the valence electrons is not properly accounted for in the AS model, the enhancement factor for the $3 d$ contribution is underestimated, which results in an almost perfect agreement between the calculated and experimental values. It should be mentioned for completeness that comparison between calculations and earlier data ${ }^{11}$ did not show such good agreement. This discrepancy may be due to differences in the measurements in Refs. 4 and 11. The two Ge detector system ${ }^{4}$ yields somewhat different results from the Ge detector-NaI detector combination used in Ref. 11, and gives slightly higher amplitudes for the core contribution in the high-momentum region.

The calculated values for $\mathrm{Nb}$ are compared with experimental data in Fig. 3. A similar comparison for Sn showed good agreement. ${ }^{6}$ The discrepancy here appears to be due to an overestimation of the $4 d$ and the $4 p$ contributions. The results for W (Fig. 4) show that the discrepancy between experiment and theory is due to the overestimation of the $4 f$ contribution. The magnitude of the discrepancy in all cases appears to be less than or about a factor of 2 . The difference between the shapes of the experimental and calculated momentum distributions is mainly due to the fact that the freeatom description is valid for the inner core electrons but not very good for the outermost ones. The calculated curves therefore agree well with the experimental ones at large momenta, but differ in shape at the smaller momenta where the dominant contribution arises from the outer core or valence electrons.

These comparisons between calculations and experimental data lead to the conclusion that the momentum density contributions of the more localized $1 s, 2 s, 2 p$, and (for elements in the fourth row and higher) $3 s$ and $3 p$ electron orbitals are well described by the current approximations in the theory. However, the shapes of the momentum density distributions due to annihilations with $3 d, 4 p, 4 d$, and $4 f$ electron levels do not agree well with the shapes of the experimental data. The partial annihilation rates for these levels may also be too high. Different functional forms for the enhancement factor have been used in the past to improve agreement between calculated and measured values of the positron lifetime. However, the positron lifetime is dominated by annihilations with valence electrons and good agreement between the calculated and measured lifetimes does not automatically imply that core electron enhancement is being taken into account correctly.

To differentiate between the momentum distributions for materials close to each other in the Periodic Table, the concept of ratio curves (i.e., the ratio of the momentum distribution of the sample material to the momentum distribution of a standard reference material) was introduced in Ref. 4, and experimental and calculated curves for a number of materials were compared. Such ratio curves contain one or more signature peaks, whose position, shape, and magnitude can be used to identify the chemical species after due account has been taken of the effect of the reference material and the resolution $^{27}$ on these peaks. Although only qualitative agreement was observed, all features observed in the experimental data were faithfully reproduced by the calculations.

The calculated values of the momentum distribution for $\mathrm{Fe}, \mathrm{Ni}, \mathrm{Cu}, \mathrm{Zn}$, and $\mathrm{Ge}$ are plotted in Fig. 5. The momentum distribution curves for $\mathrm{Ni}$ and $\mathrm{Zn}$ may not be easy to differentiate, but those of the other materials are quite distinguishable. To highlight the differences between these distributions, the ratio curves for these materials are plotted in Fig. 6 . We note that as the atomic number of the materials increases ( $\mathrm{Fe} \mathrm{27,} \mathrm{Ni} 28$, and $\mathrm{Cu} 29$ ) the position of the peak moves to higher momenta, and the height of the peak also increases. From Fig. 2 we see that for $\mathrm{Cu}$ the $\left(15<p_{L}\right.$ $<40) \times 10^{-3} m_{0} c$ region is dominated by annihilations with the $3 d$ electrons. $\mathrm{Fe}, \mathrm{Ni}$, and $\mathrm{Cu}$ have six, eight, and ten electrons in the $3 d$ shells, resulting in the highest peak for 


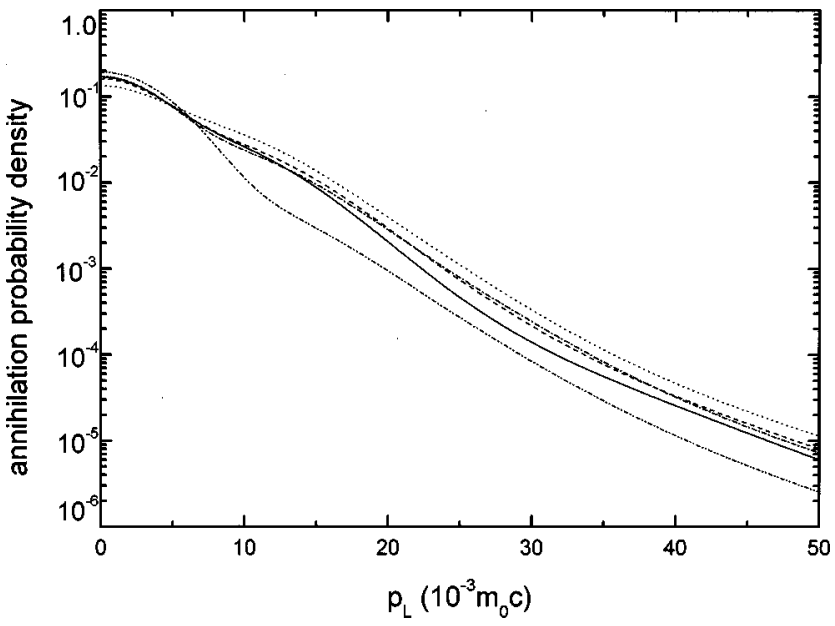

FIG. 5. The calculated annihilation probability densities of $\mathrm{Fe}$ (solid line), $\mathrm{Ni}$ (dashed line), $\mathrm{Cu}$ (dotted line), $\mathrm{Zn}$ (dot-dashed line), and Ge (dot-dot-dashed line).

$\mathrm{Cu}$. The peaks of the $\mathrm{Zn}$ and Ge curves are almost at the same point on the momentum axis, but the magnitudes are successively smaller. This decrease in magnitude is due to the different crystal structures of these materials: $\mathrm{Fe}$ is bcc, $\mathrm{Ni}$ and $\mathrm{Cu}$ are fcc, $\mathrm{Zn}$ is hcp, and $\mathrm{Ge}$ is diamondlike. In the diamond structure there is a lot of open interstitial volume, and therefore the positron wave function does not penetrate as deeply into the core region as it would in the fcc, bcc, or hcp structures.

The ratio curves for $\mathrm{Nb}, \mathrm{Mo}, \mathrm{Pd}, \mathrm{Ag}, \alpha \mathrm{Sn}$, and $\beta \mathrm{Sn}$ are plotted in Fig. 7. The systematic trends observed for these materials appear to be the same as those for Fig. 6. The positions of the second peak shift to higher momenta as the atomic number increases, the peak height increases as we go from $\mathrm{Nb}$ to $\mathrm{Pd}$, then decreases for $\mathrm{Ag}$ and $\mathrm{Sn}$. The positions of the first peak lie in the momentum region where the model is not reliable but they also follow the same trends. The effect of crystal structure is also evident, $\mathrm{Nb}$ and Mo (both bcc) are qualitatively similar, as are $\mathrm{Pd}$ and $\mathrm{Ag}$ (both are

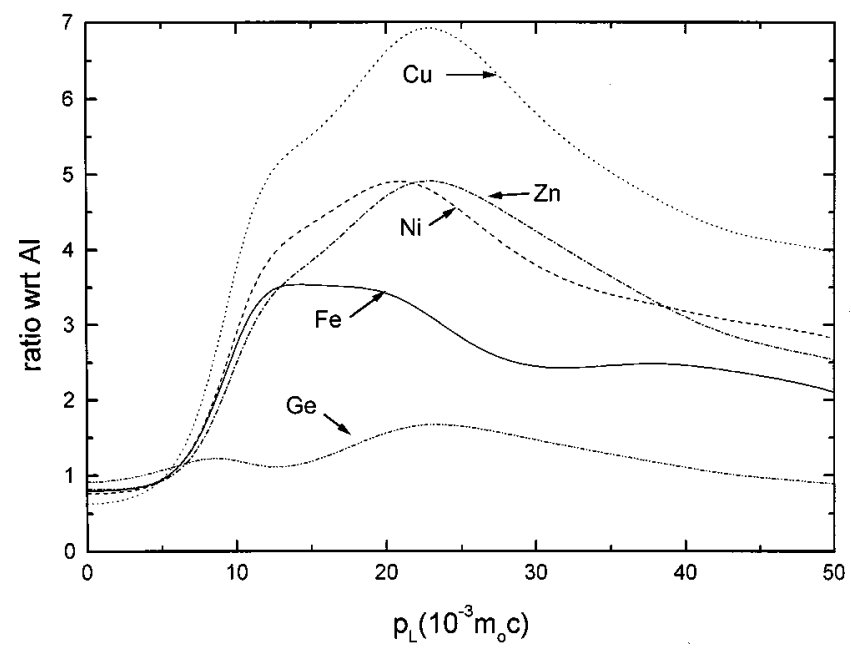

FIG. 6. Calculated ratios of the annihilation probability densities for $\mathrm{Fe}$ (solid line), $\mathrm{Ni}$ (dashed line), $\mathrm{Cu}$ (dotted line), Zn (dotdashed line), and Ge (dot-dot-dashed line) with respect to the annihilation probability density of $\mathrm{Al}$.

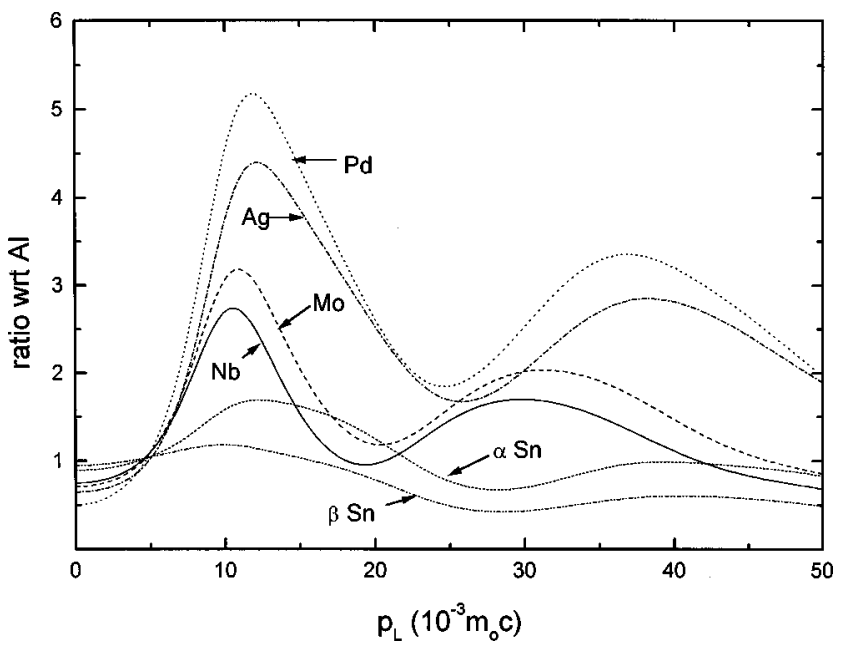

FIG. 7. Calculated ratios of the annihilation probability densities for $\mathrm{Nb}$ (solid line), Mo (dashed line), $\mathrm{Pd}$ (dotted line), Ag (dotdashed line), $\alpha$ Sn (small dashed line), and $\beta$ Sn (dot-dot-dashed line) with respect to the annihilation probability density of $\mathrm{Al}$.

fcc). $\alpha \mathrm{Sn}$, which has a diamond structure, is quite similar to Ge (Fig. 6). It is important to note that for $\alpha \mathrm{Sn}$ and $\beta$ Sn the position of the peaks is the same, only the amplitude of the peaks is different. Similar results were obtained for diamond and graphite, and for crystalline and amorphous $\mathrm{Si}^{6}$

The ratio curves for a number of lanthanides, $\mathrm{La}, \mathrm{Ce}, \mathrm{Eu}$, $\mathrm{Gd}, \mathrm{Yb}, \mathrm{W}, \mathrm{Au}$, and $\mathrm{Pb}$ are plotted in Fig. 8. As observed earlier the position of the second peak shifts toward higher momenta as the atomic number increases, the height of the peak increases with atomic number up to $\mathrm{Au}$, and then decreases for $\mathrm{Pb}$. The increase in the peak height for the lanthanides can be correlated to the increased annihilation with the $4 f$ electron level, whereas the increase for $\mathrm{W}$ and $\mathrm{Au}$ is due to the filling of the $5 d$ level. $\mathrm{Pb}$, which has completely filled $4 f$ and $5 d$ shells, has four valence electrons, $6 s^{2}$ and $6 p^{2}$, and is quite similar to $\mathrm{Sn}\left(4 d^{10} 5 s^{2} 5 p^{2}\right)$ (Fig. 7), Ge $\left(3 d^{10} 4 s^{2} 4 p^{2}\right.$ ) (Fig. 6), Si, and diamond (Ref. 6). All these

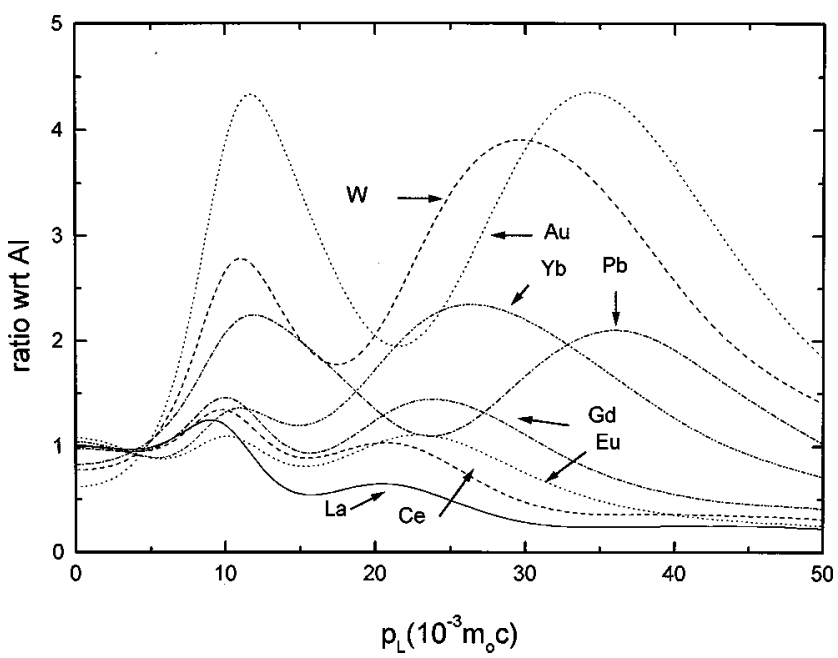

FIG. 8. Calculated ratios of the annihilation probability densities for $\mathrm{La}$ (solid line), Ce (dashed line), Eu (dotted line), Gd (dotdashed line), Yb (dot-dot-dashed line), W (dashed line), Au (dotted line), and $\mathrm{Pb}$ (dot-dashed line) with respect to the annihilation probability density of Al. 


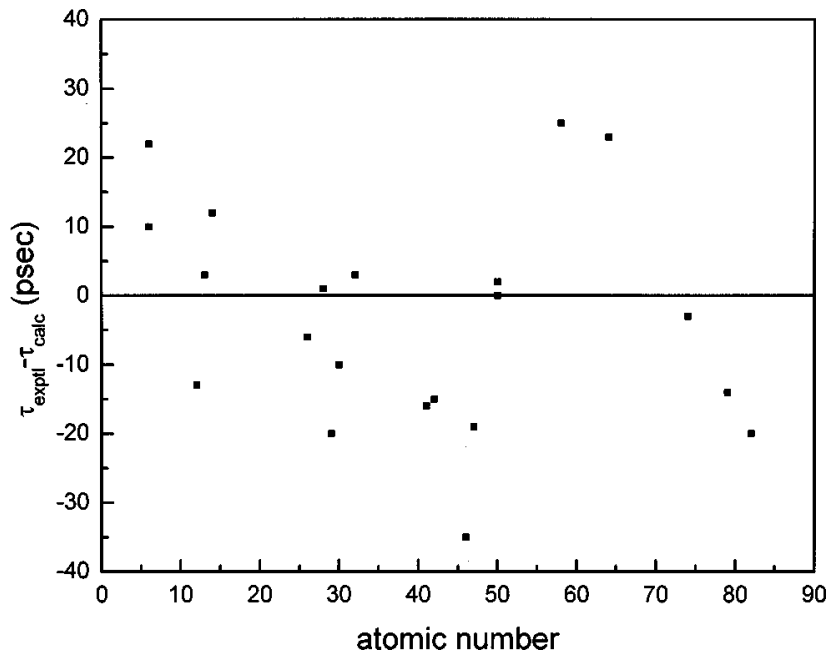

FIG. 9. The difference between the experimental and calculated lifetimes as a function of the atomic number. The experimental values are taken from Seeger (Ref. 29).

materials except $\mathrm{Pb}$ have diamond structures.

The data in Figs. 6, 7, and 8 lead to the conclusion that the ratio curves of the momentum distributions exhibit some systematic trends, like variations with atomic number, and therefore can be used to identify the chemical nature of the annihilation site. Systematic variations with atomic number have been observed for ratio curves with respect to $\mathrm{Si}$ for a number of elemental materials. ${ }^{28}$

The calculation of the positron lifetime is not the central focus of our work, but it is a useful by-product. Comparisons between the calculated and experimental values of the positron lifetime was used by Barbiellini et al. ${ }^{17}$ to determine the value of the parameter $\alpha$ used in the GGA to obtain the enhancement factors. Since we use the value of $\alpha=0.22$ used by Barbiellini et al., our calculated values are practically identical to theirs ${ }^{17}$ for $\mathrm{Al}, \mathrm{Si}, \mathrm{Fe}, \mathrm{Ni}, \mathrm{Cu}$, and $\mathrm{Ge}$. Our calculated values for a large number of elemental materials are compared to the experimental values quoted by Seeger et al. ${ }^{29}$ in Fig. 9. Apart from two isolated cases (diamond and Pd) the agreement between the calculated and measured values was found to be no worse than $\pm 15 \%$. We checked to see if the agreement between the calculated and measured values improved or deteriorated as the atomic number increased, but no such simple trend was observed.

The value of the positron lifetime is largely dominated by the partial annihilation rates of the valence electrons, resulting in practically identical values for different materials as long as the valence configuration remains unchanged. An interesting example are the lanthanides, $\mathrm{La}, \mathrm{Ce}, \mathrm{Gd}$, etc. which have similar valence configurations but different numbers of $4 f$ electrons. The measured positron lifetimes in these materials (except for $\mathrm{Eu}$ and $\mathrm{Yb}$ ), differ by less than $14 \pm 3$ ps only. ${ }^{29-31}$ However, their core momentum distributions (which are dominated by the $4 f$ contribution) are dramatically different. Hence, in this case, the momentum distribution can be used to characterize these materials whereas the positron lifetime cannot be so used.

The positron annihilation rates are a product of the charge densities of the positrons and electrons and the statedependent enhancement factors. These enhancement factors compensate for the fact that the electron wave functions are modified in the presence of the positron, and this modification is not taken into account in most calculations. The major contribution to the positron lifetime comes from annihilations with valence electrons. In spite of the fact that the atomic wave functions used in our calculations for valence electrons are not a good representation of the crystal wave functions, discrepancies between experimental and calculated values were found to be less than $\pm 15 \%$. Sterne and Kaiser $^{32}$ calculated the electron charge density by performing a self-consistent electronic-structure calculation using the LMTO method with the atomic-sphere approximation. Using two different forms of the enhancement factor, they obtained lifetimes which differ typically by \pm 5 but no more than $\pm 10 \%$ from the experimental values. They used different functional forms for the enhancement factors associated with core and valence electrons. Puska, ${ }^{23}$ also using a selfconsistent LMTO-ASA method and local-densityapproximation (LDA) enhancement factors, showed that the calculated values were in good agreement with experiment for the simple metals, but that the calculated values were consistently lower than the experimental values for the transition metals and the II-VI compound semiconductors. This discrepancy was largely due to the overestimation of the $3 d$ enhancement by the LDA, and was partially corrected by introducing the GGA. ${ }^{17}$ Our comparisons of the calculated and measured momentum densities show that enhancement for the $3 d, 4 d, 5 d$, and $4 f$ electrons is overestimated by the GGA when the fully self-consistent band structure is not used in the calculation.

Recently, an extensive study of enhancement factors was performed by Rubaszek et al. ${ }^{33}$ Although it was found that the use of a weighted density approximation gave somewhat more accurate lifetimes for some systems than the LDA or the GGA, the study did not lead to a generally applicable prescription. It should furthermore be noted that the enhancement factors used in the present work do not depend explicitly on momentum, as was the case in the early work of Kahana. This too will have an effect on a comparison between theory and experiment.

\section{CONCLUSIONS}

In this paper, calculated values of the Doppler broadening of the annihilation radiation have been presented for a large number of defect-free bulk materials. A scheme based on the method of superimposed atoms was used for these calculations. Some representative results have been compared to experimental data, and a qualitative agreement between calculated and experimental values has been demonstrated. Ratio curves have been plotted for a large number of materials, and it has been shown that these ratio curves are a good way of highlighting the differences between materials close to each other in the Periodic Table. The calculations agree qualitatively with all experimental trends observed so far, but the quantitative disagreement with the experimental data may be as large as a factor of 2 even for core electrons.

By comparing the calculated and measured Doppler broadening curves for $\mathrm{Al}, \mathrm{Cu}, \mathrm{Nb}$, and $\mathrm{W}$, we can draw some broad conclusions about our calculation methods and how these calculations can be improved. Since we are using free- 
atom electron wave functions which are better descriptions of core rather than valence electrons, we do not expect good agreement for the low-momentum region $\left[p_{L}<(20\right.$ $\left.\left.\times 10^{-3}\right) m_{0} c\right]$. The agreement between theory and experiment can be improved by performing band-structure calculations for defect-free materials. However, for materials with multiple vacancies or impurity-vacancy clusters, bandstructure calculations may not be a viable option. The major advantage of using the atomic superposition method for these calculations is its simplicity and speed, and the fact that it can reproduce, at least qualitatively, the major experimental results.

The main cause of the discrepancy in the high-momentum region between calculations and experimental data for the transition metals, noble metals, and lanthanides appears not only to be an overestimation of the $3 d, 4 d, 5 d$, and the $4 f$ contributions, but the basic shape of these contributions as well. A better understanding of the core contributions will emerge from detailed comparisons of calculated and experi- mental values in the high-momentum part of the Dopplerbroadened line shapes.

The coincidence measurements of the Doppler broadening of the annihilation radiation have primarily been used for the identification of defects in semiconductors, and for the characterization of open-volume defects in metals. The present library of fingerprints of different materials, both measured and calculated, can be extremely useful for the identification and characterization of dopant- and impurity-vacancy complexes in materials, and for the study of evolution and migration of these defects.

\section{ACKNOWLEDGMENTS}

We would like to thank M. J. Puska for various discussions and for the use of computer codes developed at the Helsinki University of Technology. This work was supported in part by the U.S. Department of Energy under Contract No. DE-AC02-98CH10886.
*Present address: Philips Research Laboratories, Prof. Holstlaan 4, 5656 AA Eindhoven, The Netherlands.

${ }^{1}$ M.J. Puska and R.M. Nieminen, Rev. Mod. Phys. 66, 841 (1994).

${ }^{2}$ P.J. Schultz and K.G. Lynn, Rev. Mod. Phys. 60, 701 (1988); K.G. Lynn, D.O. Welch, J. Throwe, and B. Nielsen, Int. Mater. Rev. 36, 1 (1991); P. Asoka-Kumar, K.G. Lynn, and D.O. Welch, J. Appl. Phys. 76, 4935 (1994).

${ }^{3}$ See reviews in Positron Spectroscopy of Solids, edited by A. Dupasquier and A. P. Mills, Jr. (IOS, Amsterdam, 1995).

${ }^{4}$ P. Asoka-Kumar, M. Alatalo, V.J. Ghosh, A.C. Kruseman, B. Nielsen, and K.G. Lynn, Phys. Rev. Lett. 77, 2097 (1996).

${ }^{5}$ J.R. MacDonald, K.G. Lynn, R.A. Boie, and M.F. Robbins, Nucl. Instrum. Methods 153, 189 (1978).

${ }^{6}$ M. Alatalo, P. Asoka-Kumar, V.J. Ghosh, B. Nielsen, K.G. Lynn, A.C. Kruseman, A. van Veen, T. Korhonen, and M.J. Puska, J. Phys. Chem. Solids 59, 55 (1998).

${ }^{7}$ M. Alatalo, H. Kauppinen, K. Saarinen, M.J. Puska, J. Mäkinen, P. Hautojärvi, and R.M. Nieminen, Phys. Rev. B 51, 4176 (1995).

${ }^{8}$ V.J. Ghosh, M. Alatalo, P. Asoka-Kumar, K.G. Lynn, and A.C. Kruseman, Appl. Surf. Sci. 116, 278 (1997).

${ }^{9}$ S. Szpala, P. Asoka-Kumar, B. Nielsen, J.P. Peng, S. Hayakawa, K.G. Lynn, and H.-J. Gossman, Phys. Rev. B 54, 4722 (1996).

${ }^{10}$ K. Saarinen, T. Laine, K. Skog, J. Mäkinen, P. Hautojärvi, K. Rakennus, P. Uusimaa, A. Salokatve, and M. Pessa, Phys. Rev. Lett. 77, 3407 (1996); T. Laine, K. Saarinen, J. Mäkinen, P. Hautojärvi, C. Corbel, L.N. Pfeiffer, and P.H. Citrin, Phys. Rev. B 54, R11 050 (1996).

${ }^{11}$ M. Alatalo, B. Barbiellini, M. Hakala, H. Kauppinen, T. Korhonen, M.J. Puska, K. Saarinen, P. Hautojärvi, and R.M. Nieminen, Phys. Rev. B 54, 2397 (1996).

${ }^{12}$ B. Barbiellini, M.J. Puska, M. Alatalo, M. Hakala, A. Harju, T. Korhonen, S. Siljamäki, T. Torsti, and R.M. Nieminen, Appl. Surf. Sci. 116, 283 (1997).

${ }^{13}$ K. Saarinen, J. Nissila, H. Kauppinen, M. Hakala, M.J. Puska, P. Hautojarvi, and C. Corbel, Phys. Rev. Lett. 82, 1883 (1999).

${ }^{14}$ P.E. Mijnarends, A.C. Kruseman, A. van Veen, H. Schut, and A. Bansil, J. Phys.: Condens. Matter 10, 10383 (1998).

${ }^{15}$ M.J. Puska and R.M. Nieminen, J. Phys. F: Met. Phys. 13, 333 (1983)
${ }^{16}$ A.P. Seitsonen, M.J. Puska, and R.M. Nieminen, Phys. Rev. B 51, 14057 (1995).

${ }^{17}$ B. Barbiellini, M.J. Puska, T. Torsti, and R.M. Nieminen, Phys. Rev. B 51, 7341 (1995); B. Barbiellini, M.J. Puska, T. Korhonen, A. Harju, T. Torsti, and R.M. Nieminen, ibid. 53, 16201 (1996).

${ }^{18}$ Calculations were performed on logarithmic grids with different numbers of mesh points. It was found that calculations on insufficiently dense grids resulted in unphysical wave function nodes and plateaus in the high-momentum part of the momentum density. The meshes used by us (25000 points) yield numerically stable values of the momentum density up to $\left(100 \times 10^{-3}\right) m_{0} c$.

${ }^{19}$ The nonrelativistic positron wave function couples only with the large components of the relativistic electron four-component spinor (Ref. 20). The small components nevertheless affect the results via the wave function normalization.

${ }^{20}$ G.J. Rozing, P.E. Mijnarends, and R. Benedek, Phys. Rev. B 43, 6996 (1991).

${ }^{21}$ A. C. Kruseman (unpublished).

${ }^{22}$ V.J. Ghosh, B. Nielsen, A.C. Kruseman, P.E. Mijnarends, A. van Veen, and K.G. Lynn, Appl. Surf. Sci. 149, 234 (1999).

${ }^{23}$ M.J. Puska, J. Phys.: Condens. Matter 3, 3455 (1991).

${ }^{24}$ M. Saito and A. Oshiyama, Phys. Rev. B 53, 7810 (1996)

${ }^{25}$ M. Hakala, M.J. Puska, and R.M. Nieminen, Phys. Rev. B 57, 7621 (1998).

${ }^{26}$ B. Barbiellini, M. Hakala, M.J. Puska, R.M. Nieminen, and A.A. Manuel, Phys. Rev. B 56, 7136 (1997).

${ }^{27}$ Recent work (Ref. 22) illustrated the large impact of the detector resolution on the position, shape, and magnitude of the peaks in the ratio curves. For ratio curves of $\mathrm{Cu}$ with respect to $\mathrm{Al}$, the peak shifts to higher momenta and decreases in magnitude when the detector resolution for the reference material $(\mathrm{Al})$ worsens (i.e., its FWHM increases). If the resolution of the detector used for the reference material measurement is kept fixed, the magnitude of the peak depends on the FWHM of the detector resolution for the material under study $(\mathrm{Cu})$. The magnitude of the peak increases as the detector resolution worsens. The sensitivity of the ratio curves to the detector resolution is much stronger than we first expected from a consideration of changes in the individual profiles. As the positions and shapes of the peaks are 
used to identify the chemical species, it is important to have a precise knowledge of the detector resolution when reporting experimental data.

${ }^{28}$ U. Myler and P.J. Simpson Phys. Rev. B 56, 14303 (1997).

${ }^{29}$ A. Seeger, F. Barnhart, and W. Bauer in Positron Annihilation, edited by L. Dorikens-Vanpraet, M. Dorikens, and D. Segers (World Scientific, Singapore, 1989), p. 275.
${ }^{30}$ I. K. MacKenzie, in Positron Solid-State Physics, edited by W. Brandt and A. Dupasquier (North-Holland, Amsterdam, 1983), p. 196.

${ }^{31}$ J.L. Rodda and M.G. Stewart, Phys. Rev. 43, 255 (1963).

${ }^{32}$ P.A. Sterne and J.H. Kaiser, Phys. Rev. B 43, 13892 (1991).

${ }^{33}$ A. Rubaszek, Z. Szotek, and W.M. Temmerman, Phys. Rev. B 58, 11285 (1998). 